\title{
Understanding Nonadherence with Hydroxychloroquine Therapy in Systemic Lupus Erythematosus
}

\author{
Lucy H. Liu, Helene B. Fevrier, Robert Goldfien, Anke Hemmerling, and Lisa J. Herrinton
}

ABSTRACT. Objective. Hydroxychloroquine (HCQ) is a cornerstone to managing systemic lupus erythematosus (SLE), yet adherence to medication is poor. We sought to measure the association of adherence with 5 "dimensions of adherence" as articulated by the World Health Organization for chronic conditions: the patient's socioeconomic status, and patient-, condition-, therapy-, and healthcare system-related factors. Our longterm goal is to generate evidence to design effective interventions to increase adherence.

Methods. The retrospective cohort study included Kaiser Permanente Northern California patients $\geq 18$ years old during 2006-2014, with SLE and $\geq 2$ consecutive prescriptions for HCQ. Adherence was calculated from the medication possession ratio and dichotomized as $<80 \%$ versus $\geq 80 \%$. Predictor variables were obtained from the electronic medical record and census data. We used multivariable logistic regression to estimate adjusted OR and 95\% CI.

Results. The study included 1956 patients. Only $58 \%$ of patients had adherence $\geq 80 \%$. In adjusted analyses, socioeconomic variables did not predict adherence. Increasing age (65-89 yrs compared with $\leq 39$ yrs: OR $1.44,95 \%$ CI $1.07-1.93)$, white race $(p<0.05)$, and the number of rheumatology visits in the year before baseline ( $\geq 3$ compared with 0 or 1 : OR $1.47,95 \%$ CI $1.18-1.83$ ) were positively associated with adherence. The rheumatologist and medical center providing care were not associated with adherence.

Conclusion. At our setting, as in other settings, about half of patients with SLE were not adherent to HCQ therapy. Differences in adherence by race/ethnicity suggest the possibility of using tailored interventions to increase adherence. Qualitative research is needed to elucidate patient preferences for adherence support. (First Release June 1 2019; J Rheumatol 2019;46:1309-15; doi:10.3899/ jrheum.180946)

Key Indexing Terms: HYDROXYCHLOROQUINE

In numerous past reports, patients with systemic lupus erythematosus (SLE) have demonstrated poor medication adherence, with about half taking $<80 \%$ of their medications

From the Department of Medicine, Kaiser Permanente Oakland Medical Center, Oakland; Department of Medicine, Division of Rheumatology, University of California, San Francisco; Division of Research, and Department of Rheumatology, Kaiser Permanente Northern California, Oakland; Bixby Center for Global Reproductive Health, Department of Obstetrics, Gynecology and Reproductive Sciences, University of California at San Francisco, San Francisco, California, USA.

L.H. Liu, MD, MPH, Department of Medicine, Kaiser Permanente Oakland Medical Center, and Department of Medicine, Division of Rheumatology, University of California, San Francisco; H.B. Fevrier, MSPH, MS, Division of Research, Kaiser Permanente Northern California; R. Goldfien, MD, Department of Rheumatology, Kaiser Permanente Northern California; A. Hemmerling, MD, PhD, MPH, Bixby Center for Global Reproductive Health, Department of Obstetrics,

Gynecology and Reproductive Sciences, University of California at San Francisco; L.J. Herrinton, PhD, Division of Research, Kaiser Permanente Northern California.

Address correspondence to L.J. Herrinton, Division of Research, Kaiser Permanente Northern California, 2000 Broadway, Oakland, California 94612, USA.E-mail: Lisa.Herrinton@kp.org

Accepted for publication January 10, 2019.

\section{SYSTEMIC LUPUS ERYTHEMATOSUS}

as instructed ${ }^{1}$. Adherence to hydroxychloroquine (HCQ) is of special interest because its side effects profile is mild, and the drug is evidenced to be highly effective in correlating directly with reduced SLE disease activity, decreased acute care use, slower progression of lupus nephritis, reduced lupus flares, and lower mortality 2,3 .

The World Health Organization (WHO) has articulated 5 "dimensions of adherence" for chronic conditions, categorizing potential barriers to adherence ${ }^{4}$. These include the patient's socioeconomic status, as well as patient-, condition-, therapy-, and healthcare system-related factors. Socioeconomic status refers to a person's wealth, education, and occupation, which reflects economic barriers to care as well as health literacy. Patient factors include beliefs and attitudes regarding treatment, which may be influenced by age and culture. Condition-related factors include comorbidities, functional limitations, and overall complexity of the disease. Therapy-related factors include the complexity of the medication regimen, including co-therapies. Health system factors include integration, care coordination, and access to care. It also

Personal non-commercial use only. The Journal of Rheumatology Copyright $\subset$ (2019. All rights reserved. 
includes physician workflows and the local context in which the physician works.

Medication adherence can be classified as primary and secondary adherence. Primary adherence is defined as whether patients filled their first prescription, whereas secondary adherence is defined as whether patients continue to fill their prescription over a long period ${ }^{5}$. Primary medication adherence is measured as a single event in time, but secondary medication adherence is a continuous measure and is of more interest in the management of chronic diseases. The reasons for poor adherence to HCQ are not well understood. Qualitative studies have cited the fear of adverse effects and patient perceptions of drug efficacy as important reasons for medication nonadherence ${ }^{6}$. Depression and complex medication regimens are common in SLE, and both are associated with poor adherence ${ }^{7}$. A recent study done in the US Medicaid population has demonstrated that an astounding $85 \%$ of patients with SLE were nonadherent to HCQ. Nonadherence was increased in younger patients as well as black and Hispanic patients ${ }^{8}$.

Our longterm goal is to develop evidence-based interventions to improve SLE patients' adherence to HCQ. Toward that goal, we conducted a retrospective study to identify factors associated with adherence. We used the WHO framework, together with US Census data and information from the health plan's electronic medical records to assess predictors of HCQ adherence in patients with SLE.

\section{MATERIALS AND METHODS}

Setting. Kaiser Permanente Northern California is a comprehensive and integrated healthcare organization with a closed pharmacy system that provides care to 3.8 million members. The region has more than 200 pharmacies available to members, located within outpatient offices and hospitals. Most members (> 95\%) obtain their medications through a Kaiser Permanente pharmacy or mail-order system. About two-thirds of patients have a simple, fixed drug copayment ranging from $\$ 5$ to $\$ 20$ (all dollars US), while others have coinsurance (e.g., 20\%), with out-of-pocket maximums varying by insurance policy. Patients can refill their medications by telephone or through the online patient portal. Our study period, using data during 2006-2014, preceded health insurance expansion under the Affordable Care Act in 2014 and the subsequent changes in health plans that Kaiser Permanente offered. Prior health plans offered individual coverage, employer-sponsored coverage, Senior Advantage Plan for Medicare-eligible patients, and Medicaid.

Study population. The population included in this retrospective cohort study was patients over 18 years of age with 2 recorded diagnosis codes for SLE [International Classification of Diseases, 9th revision (ICD-9) code 710.0], entered by a rheumatologist in an outpatient setting. We only included patients with at least 2 fills for HCQ, which is necessary for calculating medication possession ratio (MPR), our primary outcome. We required the patient to obtain the second fill within 30 days after finishing the pills included in the first fill, as assessed through the day-supply variable. We did not include patients without a refill within 30 days because those patients were more likely to have an HCQ allergy or adverse effect. The date of the second fill was used as the index date. We required patients included in the analysis to be enrolled in the health plan for at least 12 months prior to the index date to assess the patient's baseline health condition and health care use. We excluded patients with contraindications to HCQ, including retinopathy (ICD-9 codes 362.01-362.07, 362.10-362.12), toxic maculo- pathy (362.55), and allergy (995.27, 995.3, V148, V149), recorded during the 12-month period before the index date.

Data collection. Clinical data used for the study were obtained from the EPIC-based electronic medical record, established in 2005. We also used information from the 2010 census.

Our primary outcome is the rate of secondary adherence as measured by MPR, a well-established measure of medication adherence in electronic database research that has been validated in other chronic diseases ${ }^{9,10}$. MPR is defined as the number of days of dispensed medication (days-supply) divided by the total days of followup. If patients were to refill their medication sooner than the last prescription, patients can have "overadherence" where the days-supply is greater than the total days of followup, resulting in an MPR $>1$. Because of this limitation, the algorithm used to estimate MPR allowed for 7 days of stockpiling, and we did not allow the MPR to rise above 1 on any day. Followup to assess the MPR began on the index date and ended on the date of disenrollment from the health plan or the second anniversary of the index date, whichever was earlier. Adherence to HCQ was dichotomized as MPR $<80 \%$ and $\geq 80 \%$, a conventional threshold often used to define adherence in previous studies conducted on insured patient population ${ }^{10,11}$.

We used information from the 2010 US Census and from the electronic medical record to assess predictors of adherence. The US Census is a population survey collected every 10 years and provides demographic data by geography. Information on predictors was obtained during the 1-year period before the patient's second HCQ fill. The first dimension of the WHO adherence framework, socioeconomic status, was determined by linking the patient's address code to their census block (comprising about 600-3000 people) and obtaining mean values for the block-group. Variables used included median household income, percent of household income below poverty level, percent unemployment, and percent of adults who completed high school (all quartiles). Patient-related factors included age $(\leq 39,40-49$, 50-64, $\geq 65 \mathrm{yrs}$ ), sex, race/ethnicity (African American, Asian American, Hispanic, white, multiracial/other, and unknown), and language preference (English, other). Condition-related factors included the Charlson Comorbidity Index $(0,1, \geq 2)$, a composite score recording multiple comorbidities such as age, diabetes, liver disease, cardiovascular disease, chronic kidney disease, chronic obstructive pulmonary disease, stroke, and other chronic diseases ${ }^{12}$. We also examined the number of all-cause hospitalizations $(0, \geq 1)$ and the estimated glomerular filtration rate (eGFR) as an indicator of renal function. Therapy-related factors included prescriptions for SLE drugs, including prednisone (yes, no), mycophenolate mofetil (yes, no), azathioprine (yes, no), cyclophosphamide (CYC; yes, no), and angiotensin-converting enzyme (ACE) inhibitor (yes, no), as well as the number of rheumatology visits $(0-1,2,3, \geq 4)$ in the year prior to index date. We chose these medications to determine both medication complexity as well as the severity of disease, because they are used to treat renal involvement in SLE. Health systemrelated factors included the rheumatologist who prescribed the HCQ and the medical center at which the patients received their rheumatology care, an overall measure of clinical workflow, leadership, and culture.

Statistical analysis. We used chi-square tests to assess the significance of categorical variables in univariate analysis. For each adherence factor except rheumatologist and medical center, we used the SAS procedure GENMOD to perform logistic regression analysis to estimate the OR and its $95 \% \mathrm{CI}$ for the association of each factor with HCQ adherence $\geq 80 \%$. We studied crude OR by examining each independent variable one at a time. We then combined variables into an adjusted model, including variables with $p$ values $<0.2$ from the unadjusted analyses. Finally, we reduced the adjusted model by removing variables that were not associated with adherence and did not function as confounders with other variables. We used the SAS procedure GLIMMIX to perform logistic regression analysis while accounting for the random effects of rheumatologist and then medical center. This was done by using a binomial distribution with a logit link function. We assessed the fit of each model using the likelihood ratio test, and we compared models using the Akaike information criterion. A p value of 0.05 was chosen as threshold for statistical significance. All statistical analyses were conducted using SAS version 9.3 (SAS Institute Inc.). 
This study was approved by the Institutional Review Board at the Kaiser Foundation Research Institute (CN-14-2069-H).

\section{RESULTS}

The study population included 1956 patients with SLE (Figure 1). The MPR was estimated from a full 2 years of observation in $76 \%$ of patients, while the mean length of observation to calculate adherence was 21.4 months. Only $58 \%$ of patients achieved $\geq 80 \%$ HCQ adherence. Among those with adherence $\geq 80 \%$, the mean adherence was $96 \%$, while among those with adherence $<80 \%$, the mean adherence was $51 \%$.

The study population comprised $90 \%$ women, and mean age was 47 years (SD 15). Median household income at the level of the census block group averaged $\$ 78,710$ among adherent and $\$ 77,425$ among nonadherent patients. None of

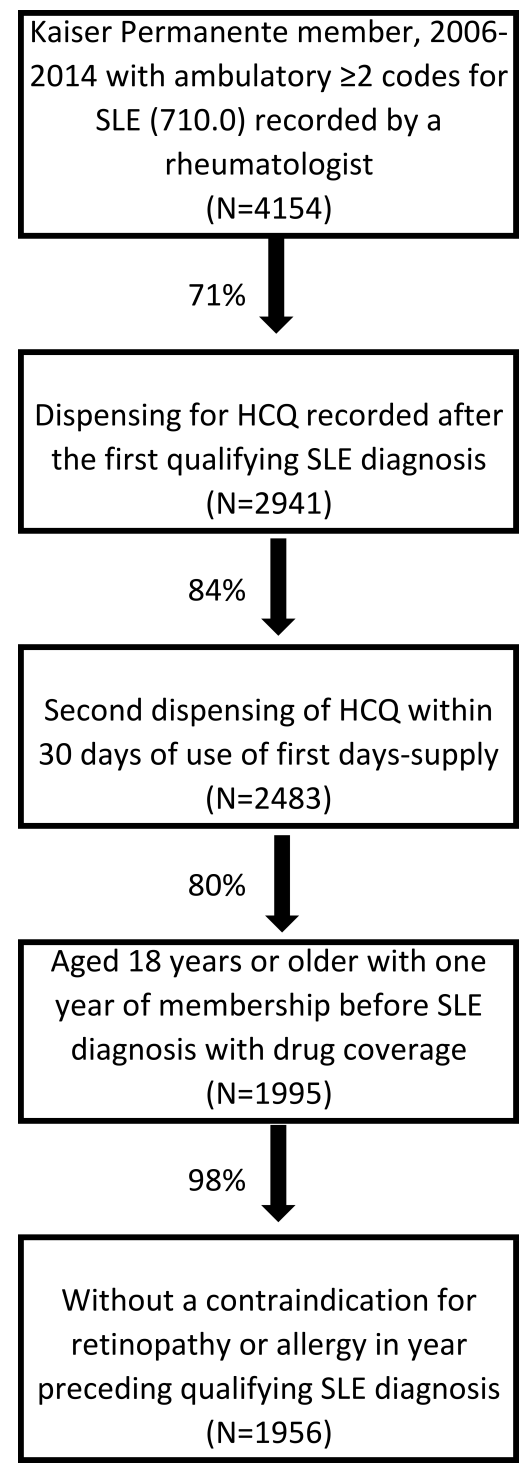

Figure 1. Cohort enrollment. SLE: systemic lupus erythematosus; HCQ: hydroxychloroquine. the 4 variables measuring socioeconomic status differed between adherent and nonadherent patients (Table 1). The adherent group was slightly older than the nonadherent group $(\mathrm{p}<0.001)$ and was disproportionately white $(\mathrm{p}<0.0001)$. Sex and language were not associated with adherence in unadjusted analyses.

We assessed 4 variables that reflect the patient's condition: Charlson comorbidity index, eGFR, number of rheumatology visits, and history of hospitalization for any cause in the year before the index date. A larger number of rheumatology visits and eGFR of $60-89$ (vs $\geq 90) \mathrm{ml} / \mathrm{min} / 1.73 \mathrm{~m}^{2}$ were associated with better adherence. The Charlson index and a history of hospitalization were not associated with adherence to a significant degree. Regarding therapy, only 3 patients used CYC, and therefore we did not further consider this drug. Mycophenolate mofetil was somewhat inversely associated with the odds of adherence (OR 0.81, 95\% CI 0.64-1.03). The use of prednisone and ACE inhibitor were not associated with adherence. Regarding healthcare system factors, compared to the average medical center, the odds of adherence $\geq 80 \%$ at the medical center with the best adherence were 1.13 (95\% CI 0.91-1.86), while at the medical center with the worst adherence, the odds were 0.84 (95\% CI 0.67-1.17; p > 0.05). The rheumatologist was not associated with adherence $(\mathrm{p}>0.05)$.

The adjusted analysis included age, race/ethnicity, and the number of rheumatology visits (Table 2). Increasing age (65-89 yrs compared with $\leq 39$ yrs: OR $1.44,95 \%$ CI $1.07-1.93)$, white race $(\mathrm{p}<0.05)$, and the number of rheumatology visits in the year before baseline ( $\geq 3$ compared with 0 or 1: OR 1.47, 95\% CI 1.18-1.83) were associated with higher adherence $\geq 80 \%$. Compared to white patients, the OR for $\geq 80 \%$ adherence was 0.74 (95\% CI 0.56-0.97) for African American patients, 0.76 (95\% CI 0.59-0.99) for Asian/Pacific Islander patients, and 0.59 (95\% CI 0.44-0.77) for Hispanic patients. We also compared adjusted analyses with and without eGFR, which ultimately was removed from the adjusted model because it was not significantly associated with adherence after accounting for age, race, and the number of rheumatology visits. Finally, we assessed whether the random effects of rheumatologist and medical center improved the fit of the adjusted model, but they did not (both $\mathrm{p}>0.05$ ).

\section{DISCUSSION}

In a community-based population with insurance coverage, relatively low drug copayments, and a high level of care coordination, only $58 \%$ of patients with SLE had $\geq 80 \%$ adherence to HCQ. Factors associated with adherence included age, race/ethnicity, and the number of rheumatology visits in the previous year, a marker of SLE severity. Our findings are consistent with previous studies that showed that age, race, and SLE severity were associated with medication adherence $^{1,2,6,7,13}$.

The study included patients who differed broadly in their

Personal non-commercial use only. The Journal of Rheumatology Copyright $\odot$ 2019. All rights reserved 
Table 1. Crude OR and 95\% CI for associations of 4 WHO dimensions of adherence with adherence $\geq 80 \%$ in 1956 Kaiser Permanente patients with SLE and 2 recent prior fills of hydroxychloroquine, Northern California, 2006-14.

\begin{tabular}{|c|c|c|c|c|c|}
\hline Characteristics & $\begin{array}{c}\text { Adherence } \geq 80 \%, \\
\mathrm{n}=1132\end{array}$ & $\begin{array}{c}\text { Adherence }<80 \%, \\
\mathrm{n}=824\end{array}$ & Crude OR & $95 \% \mathrm{CI}$ & $\mathrm{p}$ \\
\hline \multicolumn{6}{|c|}{ Socioeconomic status (block-group) } \\
\hline $15-54$ & 26.9 & 28.7 & 1.00 & Ref. & \\
\hline $55-74$ & 23.1 & 23.7 & 1.21 & $0.94-1.56$ & 0.13 \\
\hline $75-99$ & 24.7 & 25.3 & 1.04 & $0.81-1.33$ & 0.75 \\
\hline $100-250$ & 25.3 & 22.3 & 1.04 & $0.81-1.34$ & 0.74 \\
\hline $3.1-6.5$ & 25.9 & 23.5 & 1.03 & $0.80-1.33$ & 0.80 \\
\hline $6.6-12.7$ & 24.7 & 25.9 & 0.99 & $0.77-1.28$ & 0.96 \\
\hline $12.8-100$ & 24.9 & 25.1 & 1.15 & $0.89-1.48$ & 0.30 \\
\hline \multicolumn{6}{|l|}{ Percent unemployed } \\
\hline $0-3.7$ & 26.2 & 24.8 & 1.00 & Ref. & \\
\hline $3.8-5.4$ & 25.0 & 25.6 & 0.90 & $0.70-1.15$ & 0.40 \\
\hline $20-28$ & 30.1 & 31.8 & 0.90 & $0.71-1.14$ & 0.37 \\
\hline $29-59$ & 21.9 & 19.7 & 0.87 & $0.67-1.13$ & 0.30 \\
\hline \multicolumn{6}{|l|}{ Patient-related factors } \\
\hline \multicolumn{6}{|l|}{ Age, yrs } \\
\hline$\leq 39$ & 29.4 & 34.2 & 1.00 & Ref. & \\
\hline $40-49$ & 21.0 & 26.6 & 0.92 & $0.72-1.17$ & 0.50 \\
\hline $50-64$ & 32.0 & 25.8 & 1.44 & $1.14-1.82$ & 0.00 \\
\hline $65-89$ & 17.6 & 13.4 & 1.53 & $1.16-2.03$ & 0.00 \\
\hline \multicolumn{6}{|l|}{ Sex } \\
\hline Male & 10.5 & 9.0 & 1.00 & Ref. & \\
\hline & 89.5 & 91.0 & 0.84 & $0.62-1.14$ & 0.26 \\
\hline \multicolumn{6}{|l|}{ Race/ethnicity } \\
\hline African American & 15.2 & 16.9 & 0.68 & $0.52-0.89$ & $<0.01$ \\
\hline \multicolumn{6}{|l|}{ Charlson comorbidity index } \\
\hline 0 & 83.6 & 84.5 & 1.00 & Ref. & \\
\hline 1 & 9.2 & 6.9 & 1.34 & $0.96-1.88$ & 0.09 \\
\hline$\geq 2$ & 7.2 & 8.6 & 0.85 & $0.61-1.18$ & 0.34 \\
\hline \multicolumn{6}{|l|}{ Rheumatology visits } \\
\hline $0-1$ & 24.0 & 29.6 & 1.00 & Ref. & \\
\hline 2 & 26.3 & 25.1 & 1.29 & $1.01-1.65$ & 0.04 \\
\hline$\geq 3$ & 49.7 & 45.3 & 1.35 & $1.09-1.68$ & 0.01 \\
\hline \multicolumn{6}{|l|}{ Hospitalization for any cause } \\
\hline No & 81.6 & 82.0 & 1.00 & Ref. & \\
\hline Yes & 18.4 & 18.0 & 1.03 & $0.81-1.30$ & 0.82 \\
\hline \multicolumn{6}{|l|}{$\mathrm{eGFR}, * * \mathrm{ml} / \mathrm{min} / 1.73 \mathrm{~m}^{2}$} \\
\hline $30-59$ & 0.7 & 1.5 & 0.53 & $0.22-1.32$ & 0.17 \\
\hline $60-89$ & 29.1 & 21.1 & 1.52 & $1.23-1.89$ & 0.0001 \\
\hline$\geq 90$ & 70.2 & 77.4 & 1.00 & Ref. & \\
\hline Therapy-related factors in the & & & & & \\
\hline Medications (yes vs no) & & & & & \\
\hline Prednisone & 58.0 & 56.9 & 1.05 & $0.87-1.26$ & 0.62 \\
\hline Mycophenolate mofetil & 15.6 & 18.5 & 0.81 & $0.64-1.03$ & 0.09 \\
\hline Azathioprine & 11.8 & 11.5 & 1.03 & $0.78-1.36$ & 0.83 \\
\hline ACE inhibitor & 20.1 & 18.3 & 1.12 & $0.89-1.41$ & 0.32 \\
\hline
\end{tabular}

Values are \% unless otherwise specified. * Includes multiracial, Native American, and unknown. ** Missing for 65 patients. WHO: World Health Organization; SLE: systemic lupus erythematosus; eGFR: estimated glomerular filtration rate; ACE: angiotensin-converting enzyme. 
Table 2. Adjusted OR and 95\% CI for associations of patient- and condition-related factors* with adherence $\geq 80 \%$ in 1956 Kaiser Permanente patients with SLE and 2 recent prior fills of hydroxychloroquine, Northern California, 2006-14.

\begin{tabular}{lccc}
\hline Variables & Adjusted OR & $95 \%$ CI & $\mathrm{p}$ \\
\hline Age, yrs & & & \\
$\quad \leq 39$ & 1.00 & Ref. & \\
$40-49$ & 0.92 & $0.72-1.18$ & 0.51 \\
$50-64$ & 1.39 & $1.09-1.76$ & 0.01 \\
65-89 & 1.44 & $1.07-1.93$ & 0.02 \\
Race & & & \\
African American & 0.74 & $0.56-0.97$ & 0.03 \\
Asian/Pacific Islander & 0.76 & $0.59-0.99$ & 0.04 \\
Hispanic & 0.59 & $0.44-0.77$ & $<0.001$ \\
White & 1.00 & Ref. & \\
Other/unknown & 0.67 & $0.49-0.92$ & 0.01 \\
No. rheumatology visits & & & \\
0-1 & 1.00 & Ref. & \\
2 & 1.30 & $1.01-1.67$ & 0.04 \\
$\geq 3$ & 1.47 & $1.18-1.83$ & $<0.001$ \\
\hline
\end{tabular}

* Other factors in Table 1 were not associated with adherence to a significant degree in the adjusted model. The rheumatologist and the medical center also were not associated with adherence to a significant degree in the adjusted model. SLE: systemic lupus erythematosus.

socioeconomic status, but in this population with health insurance, socioeconomic status was not an important predictor of adherence. This likely reflects adequate access to medication in our integrated healthcare system. In an integrated healthcare system, patients have increased physical access to medication because clinics are in close proximity to pharmacies, and refills can be mailed directly to patients. In contrast, Feldman and colleagues' study of Medicaid patients with SLE found that patients who were nonadherent to HCQ had lower socioeconomic status than adherent patients ${ }^{2}$. One important socioeconomic factor is the ability to pay for medications. In our patient population, financial barriers are likely limited given that the average copay for medication is $<\$ 20$ for the majority of the patients. In a quasi-experimental study of Veteran's Administration patients, Doshi and colleagues observed that co-pay was inversely related to adherence ${ }^{14}$. Notwithstanding, among patients with a zero copayment, $40 \%$ were nonadherent ${ }^{14}$. HCQ is one of the less expensive medications used in rheumatology, yet adherence to HCQ is lower than adherence to biologic agents, which are costlier ${ }^{15}$. Interventions for improving medication adherence must expand beyond increasing financial accessibility.

HCQ is a medication with mild side effects that takes effect over several weeks. This may mislead patients to believe that the medication is not effective. A patient's belief in the efficacy of medication has been shown to be integral to adherence, and patients who do not experience an immediate clinical effect from a drug may not take it, regardless of other factors. Patients who have more frequent followup visits with their rheumatologists are more likely to be adherent to HCQ. Close followup provides an opportunity for providers to forge a therapeutic alliance with their patients and reinforce the importance of medication adherence. Though our study did not record remote encounters, there is some evidence that online interactions through Web-based education modules and social media group participation may improve HCQ adherence, particularly in younger patient populations ${ }^{16}$. In one study, cellular text messaging helped improved adherence to office visits but not HCQ adherence in patients with pediatric-onset SLE ${ }^{17}$. FaceTime has also been used successfully to enforce directly observed therapy remotely, increasing adherence to mycophenolate mofetil in pediatric patients with $\mathrm{SLE}^{18}$. Studies are under way to explore the use of mobile health technologies in other chronic diseases such as hypertension ${ }^{19,20,21}$, diabetes ${ }^{20,22}$, cancer treatment ${ }^{23}$, and human immunodeficiency virus ${ }^{24}$, and the findings of these studies may be applicable to the SLE population in enhancing medication adherence.

Patients who are sicker are more likely to be adherent. The patients in our community-based study have relatively few comorbidities and intact renal function, and one reason for the relatively low adherence that we observed may be the low severity or activity of SLE. Neurologic manifestations of SLE often include depression and cognitive impairment, which are not measured by the Charlson comorbidity index alone. Severity of depression in patients with SLE has been shown to strongly affect adherence ${ }^{25}$. Although not within the scope of our study, it would have been interesting to examine the effect of specific SLE disease activity on adherence.

A limitation of the study was the use of the MPR in place of more direct measures such as pill count, electronic lid monitoring, or blood level of HCQ. While MPR and other measures such as proportion of days covered (PDC) are often used to measure medication adherence based on claims data, they preclude the evaluation of primary medication adherence because they can only be calculated if at least 2 prescriptions have been filled. In one study, Tamblyn and colleagues found that up to one-third of patients in the primary care setting do not fill their new prescription, highlighting the need to understand primary as well as secondary adherence ${ }^{26}$. MPR and PDC also exclude patients who filled their first prescription, but then failed to fill their second prescription, which will likely underestimate the rate of secondary medication adherence. More recent studies have demonstrated the utility of HCQ blood level to monitor medication adherence and correlation with SLE flare $27,28,29$. Future studies on medication adherence in HCQ can be strengthened by measuring blood level rather than using MPR.

Our study also did not examine the dosing schedule of HCQ as related to adherence, which can vary by weight and renal function. Although it is well known that increasing dosing frequency impairs adherence ${ }^{30}$, our analysis would have been complicated by changes in dosing schedule during Personal non-commercial use only. The Journal of Rheumatology Copyright @ 2019 . All rights reserved. 
the study period. The strengths of our study included access to comprehensive information, the integrated setting in which certain sources of variation were minimized, and the large and diverse study population. Despite the integrated setting, we found a high level of nonadherence with HCQ therapy.

Medication adherence is a major public health problem in the management of SLE and other chronic illnesses and is thought to cost the US healthcare system an excess of $\$ 100$ billion to $\$ 300$ billion a year ${ }^{31}$. Given this burden, it is critical to determine contributing causes and to develop and create evidence for tailored interventions to improve adherence $^{32}$. Research is needed to better understand the role of patient beliefs and attitudes, the patient-physician relationship and communication, and health literacy. We recommend mixed method and qualitative approaches to learn why patients are not taking their medications.

\section{REFERENCES}

1. Koneru S, Kocharla L, Higgins GC, Ware A, Passo MH, Farhey YD, et al. Adherence to medications in systemic lupus erythematosus. J Clin Rheumatol 2008;14:195-201.

2. Feldman CH, Yazdany J, Guan H, Solomon DH, Costenbader KH. Medication nonadherence is associated with increased subsequent acute care utilization among medicaid beneficiaries with systemic lupus erythematosus. Arthritis Care Res 2015;67:1712-21.

3. Ruiz-Irastorza G, Ramos-Casals M, Brito-Zeron P, Khamashta MA. Clinical efficacy and side effects of antimalarials in systemic lupus erythematosus: a systematic review. Ann Rheum Dis 2010;69:20-8.

4. World Health Organization. Adherence to long-term therapies: evidence for action. [Internet. Accessed March 28, 2019.] Available from: www.who.int/chp/knowledge/publications/adherence_report/en

5. Raebel MA, Schmittdiel J, Karter AJ, Konieczny JL, Steiner JF. Standardizing terminology and definitions of medication adherence and persistence in research employing electronic databases. Med Care 2013;51:S11-21.

6. Costedoat-Chalumeau N, Pouchot J, Guettrot-Imbert G, Le Guern V, Leroux G, Marra D, et al. Adherence to treatment in systemic lupus erythematosus patients. Best Pract Res Clin Rheumatol 2013;27:329-40.

7. Petri M, Perez-Gutthann S, Longenecker JC, Hochberg M. Morbidity of systemic lupus erythematosus: role of race and socioeconomic status. Am J Med 1991;91:345-53.

8. Feldman CH, Collins J, Zhang Z, Subramanian SV, Solomon DH, Kawachi I, et al. Dynamic patterns and predictors of hydroxychloroquine nonadherence among Medicaid beneficiaries with systemic lupus erythematosus. Semin Arthritis Rheum 2018;48:205-13.

9. Márquez-Contreras E, López García-Ramos L, Martell-Claros N, Gil-Guillen VF, Márquez-Rivero S, Pérez-López E, et al. Validation of the electronic prescription as a method for measuring treatment adherence in hypertension. Patient Educ Couns 2018;101:1654-60.

10. Andrade SE, Kahler KH, Frech F, Chan KA. Methods for evaluation of medication adherence and persistence using automated databases. Pharmacoepidemiol Drug Saf 2006;15:565-74.

11. Karve S, Cleves MA, Helm M, Hudson TJ, West DS, Martin BC. Good and poor adherence: optimal cut-point for adherence measures using administrative claims data. Curr Med Res Opin 2009;25:2303-10.

12. Deyo RA, Cherkin DC, Ciol MA. Adapting a clinical comorbidity index for use with ICD-9-CM administrative databases. J Clin Epidemiol 1992;45:613-9.
13. Mehat $\mathrm{P}$, Atiquzzaman M, Esdaile JM, Aviña-Zubieta A, De Vera MA. Medication nonadherence in systemic lupus erythematosus: a systematic review. Arthritis Care Res 2017;69:1706-13.

14. Doshi JA, Zhu J, Lee BY, Kimmel SE, Volpp KG. Impact of a prescription copayment increase on lipid-lowering medication adherence in veterans. Circulation 2009;119:390-7.

15. Tkacz J, Ellis L, Bolge SC, Meyer R, Brady BL, Ruetsch C. Utilization and adherence patterns of subcutaneously administered anti-tumor necrosis factor treatment among rheumatoid arthritis patients. Clin Ther 2014;36:737-47.

16. Scalzi LV, Hollenbeak CS, Mascuilli E, Olsen N. Improvement of medication adherence in adolescents and young adults with SLE using web-based education with and without a social media intervention, a pilot study. Pediatr Rheumatol Online J 2018;16:18.

17. Ting TV, Kudalkar D, Nelson S, Cortina S, Pendl J, Budhani S, et al. Usefulness of cellular text messaging for improving adherence among adolescents and young adults with systemic lupus erythematosus. J Rheumatol 2012;39:174-9.

18. Hui Yuen JS. Usefulness of FaceTime to improve medication adherence in adolescents and young adults with systemic lupus erythematosus: a case series. Rheumatology: Current Research [Internet. Accessed March 28, 2019.] Available from: www.omicsonline.org/open-access/usefulness-of-facetime-toimprove-medication-adherence-in-adolescents-andyoung-adultswith-systemic-lupus-erythematosus-a-case-series-2161-1149-10001 53.php?aid $=54701$

19. Skolarus LE, Cowdery J, Dome M, Bailey S, Baek J, Byrd JB, et al. Reach out churches: a community-based participatory research pilot trial to assess the feasibility of a mobile health technology intervention to reduce blood pressure among African Americans. Health Promot Pract 2018;19:495-505.

20. Huo X, Spatz ES, Ding Q, Horak P, Zheng X, Masters C, et al Design and rationale of the Cardiovascular Health and Text Messaging (CHAT) Study and the CHAT-Diabetes Mellitus (CHAT-DM) Study: two randomised controlled trials of text messaging to improve secondary prevention for coronary heart disease and diabetes. BMJ Open 2017;7:e18302.

21. Chow CK, Thiagalingam A, Santo K, Kok C, Thakkar J, Stepien S, et al. TEXT messages to improve MEDication adherence and Secondary prevention (TEXTMEDS) after acute coronary syndrome: a randomised clinical trial protocol. BMJ Open 2018;8:e019463.

22. Sarayani A, Mashayekhi M, Nosrati M, Jahangard-Rafsanjani Z, Javadi M, Saadat N, et al. Efficacy of a telephone-based intervention among patients with type-2 diabetes; a randomized controlled trial in pharmacy practice. Int J Clin Pharm 2018; 40:345-53

23. Wu YP, Linder LA, Kanokvimankul P, Fowler B, Parsons BG, Macpherson CF, et al. Use of a smartphone application for prompting oral medication adherence among adolescents and young adults with cancer. Oncol Nurs Forum 2018;45:69-76.

24. Britto MT, Rohan JM, Dodds CM, Byczkowski TL. A randomized trial of user-controlled text messaging to improve asthma outcomes: a pilot study. Clin Pediatr 2017;56:1336-44.

25. Julian LJ, Yelin E, Yazdany J, Panopalis P, Trupin L, Criswell LA, et al. Depression, medication adherence, and service utilization in systemic lupus erythematosus. Arthritis Rheum 2009;61:240-6.

26. Tamblyn R, Eguale T, Huang A, Winslade N, Doran P. The incidence and determinants of primary nonadherence with prescribed medication in primary care: a cohort study. Ann Intern Med 2014;160:441-50.

27. Costedoat-Chalumeau N, LE Guern V, Piette JC. Routine hydroxychloroquine blood concentration measurement in systemic lupus erythematosus reaches adulthood. J Rheumatol 2015; 42:1997-9. 
28. Costedoat-Chalumeau N, Houssiau F, Izmirly P, Le Guern V, Navarra S, Jolly M, et al. A prospective international study on adherence to treatment in 305 patients with flaring SLE: assessment by drug levels and self-administered questionnaires. Clin Pharmacol Ther 2018;103:1074-82.

29. Durcan L, Clarke WA, Magder LS, Petri M. Hydroxychloroquine blood levels in systemic lupus erythematosus: clarifying dosing controversies and improving adherence. J Rheumatol 2015; 42:2092-7.
30. Coleman CI, Limone B, Sobieraj DM, Lee S, Roberts MS, Kaur R, et al. Dosing frequency and medication adherence in chronic disease. J Manag Care Pharm 2012;18:527-39.

31. Iuga AO, McGuire MJ. Adherence and health care costs. Risk Manag Healthc Policy 2014;7:35-44.

32. Viswanathan M, Golin CE, Jones CD, Ashok M, Blalock S, Wines $\mathrm{RC}$, et al. Closing the quality gap: revisiting the state of the science (vol. 4: medication adherence interventions: comparative effectiveness). Evid Rep Technol Assess (Full Rep) 2012;1-685. 